\title{
The 4-hook anchor coaxial needle with scaled suture is superior to the double spring coil for preoperative localization
}

\author{
Zhi-Ming Chen ${ }^{1 \#}$, Jia-Yang Xu ${ }^{2 \#}$, Wen-Qing Cai ${ }^{1}$, Fa-Chao Liao ${ }^{1}$, Shan-Qi Huo ${ }^{1}$, Jin-Wei Yang ${ }^{3}$, Jun Peng $^{4}$ \\ ${ }^{1}$ Department of Radiology, the First People's Hospital of Yunnan Province, the Affiliated Hospital of Kunming University of Science and Technology, \\ Kunming, China; ${ }^{2}$ Medical School of Kunming University of Science and Technology, Kunming, China; ${ }^{3}$ The Second Department of General \\ Surgery, the First People's Hospital of Yunnan Province, the Affiliated Hospital of Kunming University of Science and Technology, Kunming, China; \\ ${ }^{4}$ Department of Thoracic Surgery, the First People's Hospital of Yunnan Province, the Affiliated Hospital of Kunming University of Science and \\ Technology, Kunming, China \\ Contributions: (I) Conception and design: ZM Chen, JW Yang, J Peng; (II) Administrative support: ZM Chen, JW Yang, J Peng; (III) Provision of \\ study materials or patients: ZM Chen, J Peng; (IV) Collection and assembly of data: WQ Cai, FC Liao, SQ Huo, JY Xu; (V) Data analysis and \\ interpretation: ZM Chen, JY Xu; (VI) Manuscript writing: All authors; (VII) Final approval of manuscript: All authors. \\ "These authors contributed equally to this work. \\ Correspondence to: Jun Peng. Department of Thoracic Surgery, the First People's Hospital of Yunnan Province, the Affiliated Hospital of Kunming \\ University of Science and Technology, Kunming, China. Email: 389647518@qq.com; Jin-Wei Yang. The Second Department of General Surgery, \\ the First People's Hospital of Yunnan Province, the Affiliated Hospital of Kunming University of Science and Technology, Kunming, China. Email: \\ caohede@163.com.
}

Background: Preoperative localization of small size pulmonary nodules is challenging, but it is necessary for surgical resection of early lung cancer. As a new device for preoperative localization, the 4-hookanchor coaxial needle with scaled suture was tentatively applied in our department to improve the effect of preoperative localization. However, double spring coil, as a proven positioning technology, used to be our preferred method in the past. We did a retrospective single-centre research driven by the interest on which one should be the first choice for preoperative localization among these two approaches.

Methods: We performed a retrospective analysis on 100 patients undergoing surgery with the new coaxial needle from 2019 to 2020, and 98 patients undergoing double spring coil from 2017 to 2019. The duration of localization, success rate, operation time, intraoperative bleeding, and positioning-related complications of these two groups of patients were examined in this study.

Results: There were no significant differences between the two groups of patients in terms of the success rate. However, the new coaxial needle seemed to be able to shorten the duration of preparative localization and operation time by accelerating the efficiency of exploring small nodules intraoperatively, and also decreased the risk of positioning-related pneumothorax and pulmonary hemorrhage. The logistic analysis indicated that the puncture depth was an independent risk factor for overall complications. Meanwhile, previous lung diseases and positioning time were independent risk factors for pneumothorax, besides pneumorrhagia and depth of penetration as well.

Conclusions: The new coaxial needle can save time for both radiologists and thoracic surgeons, while reducing the risk of positioning-related complications. We support its application clinically instead of the double spring coil.

Keywords: Four-hook-anchor coaxial needle with scaled suture; double spring coil; preoperative localization; small size pulmonary nodules

Submitted Apr 30, 2021. Accepted for publication Jul 19, 2021.

doi: $10.21037 /$ jtd-21-984

View this article at: https://dx.doi.org/10.21037/jtd-21-984 


\section{Introduction}

With low dose computed tomography (CT) being widely used in lung screening, an increasing number of small-sized pulmonary nodules are being diagnosed (1). The majority of isolated pulmonary nodules are malignant, and thus, accurate technology is required for positioning and excision $(2,3)$. Diagnosis with percutaneous puncture biopsy is accurate in most situations, but some tumor nodules are too small to be extracted and pathologically examined from the wholly removed pulmonary tissue. This may result in unintended diagnoses, and pathological slice observation is still the gold standard (4). On the other hand, the promotion of video-assisted thoracic surgery (VATS) limits the use of the intraoperative probe, and the location of small-sized nodules is time-consuming for surgeons without preoperative localization (5). Preoperative localization is crucial, and radiologists have devoted themselves to developing localization technologies, such as methylene blue staining, hookwire positioning needle, double spring coil, etc. (6). However, various problems have arisen in the application of the above technologies (7). In this study, our team aimed to preoperatively position small-sized nodules with a new device, the 4-hook anchor coaxial needle with scaled suture, and compared its effectiveness and complications with the double spring coil. We present the following article in accordance with the STROBE reporting checklist (available at https://dx.doi.org/10.21037/jtd-21-984).

\section{Methods}

\section{General data}

We performed a retrospective analysis on patients undergoing thoracic surgery and examined the duration of localization, success rate, operation time, intraoperative bleeding, and positioning-related complications. Ethical approval for the study was granted by the Ethics Review Committee of the First People's Hospital of Yunnan Province, and informed consent was obtained from the patients before surgery. The study was conducted in accordance with the Declaration of Helsinki (as revised in 2013). All samples were collected from patients who underwent thoracoscopic anatomical pneumonectomy at the First People's Hospital of Yunnan Province. We communicate with patients in the consulting room and interventional room and get their consent. Finally we collected 100 cases of preoperative localization with the new coaxial needle and 98 cases of preoperative localization with the double spring coil from 2017 to 2020. These patients were divided into a Needle group and a Coil group accordingly, and there were no statistical differences in age $(\mathrm{t}=0.807, \mathrm{P}=0.420)$ and sex $\left(\chi^{2}=0.485, \mathrm{P}=0.486\right)$ between the two groups. Because patients retained the right of independent choice of the treatment plan, it was not able to do a random experiment under the double-blind principle. We did the certain localization method as the patients requirement. However, a random sampling was taken among the patients with similar size of pulmonary nodules.

\section{Inclusion criteria}

(I) Patients with a family history of lung cancer or lived in a high-risk area;

(II) Patients with a subjective desire for surgical treatment;

(III) The size of the nodule was $\leq 20 \mathrm{~mm}$;

(IV) The CT image indicated ground-glass nodule (GGN) or part solid nodule (PSN);

(V) Patients with no surgical contraindications.

\section{Exclusion criteria}

(I) Patients with basic diseases that would increase the risk of positioning procedures, such as a moderate amount (or more) of pleural effusion, previous damage to pulmonary tissue, severe emphysema, pulmonary bullae, severe pulmonary fibrosis, and so on;

(II) Patients with recurrent chronic lung infections or systemic sepsis;

(III) Patients with severe coagulation dysfunction;

(IV) Patients who failed to cooperate with the procedures;

(V) Patients who were assessed as not temporarily indispensable for surgical treatments by thoracic surgeons.

\section{Instruments and materials}

Siemens SOMATOM Perspective 128 spiral CT (Germany) was used. The locating instrument in the Needle group was the 4-hook anchor coaxial needle with scaled suture (Ningbo Sheng Jie Kang Biological Technology Co., Ltd., Ningbo, China). This new device is composed of a $19-\mathrm{G}$ coaxial needle, tri-colored suture, 4-hook anchor, pusher, release buckle, and protection tube. The coaxial needle and protection tube are made of medical-grade stainless steel. The tri-colored suture is essentially dyed nonabsorbable medical suture, and the different colors indicate 
different depths in the localization procedure. The 4-hook anchor is made of nickel titanium memory alloy wire. The particular "four-claw" shape facilitates reliable fixing on lung tissue. In the Coil group, a 0.018-in micro spring coil (specifications: $8 \mathrm{~mm} \times 2 \mathrm{~mm}, 6 \mathrm{~mm} \times 2 \mathrm{~mm}, 5 \mathrm{~mm}$ $\times 2 \mathrm{~mm}$; Cook Company, USA) and a percutaneous transhepatic cholangiography (PTC) interventional puncture needle $(21 \mathrm{G} \times 200$ mm; Hachiko Co., Tel Aviv, Israel) were used.

\section{Methods}

\section{Needle group}

We placed patients in the appropriate postures to achieve satisfactory scanning images. The localization was performed in the operation room, and each patient underwent at least four CT scans. The first scan was performed to ensure the position, shape, size, and density of the nodule, which was marked on the lung surface, while determining its anatomically adjacent relationships. Next, we designed a proper puncture path by calculating the expected depth and angle of the needle. Following the application of anesthesia by the anesthesiologists, the needle was pushed to $5 \mathrm{~mm}$ around the lesion along the predesigned path. In the third scan, we removed the card clasp of the coaxial needle, which was in the expected position, and pushed the pusher, thereby releasing the 4-hook anchor near the edge of the nodule. Later, we removed the pusher and the protection tube, and measured the distance from the tip of the coaxial needle to the visceral pleura. The coaxial needle was pulled back to the pleural cavity according to the measured distance, and the pusher was inserted into the coaxial needle again to remove the tri-colored suture. The final scan helped us to observe whether the surgery was successful and whether there were any complications. Subsequently, we withdrew the coaxial needle and pusher, and then addressed any complications. Pneumonectomy was performed within 2 days of preoperative localization.

\section{Coil group}

The first two scans were similar to the Needle group. In the third scan, the puncture needle was placed near the edge of the nodule, and a suitable spring coil $(5 \mathrm{~mm} \times 2 \mathrm{~mm}$, $4 \mathrm{~mm} \times 2 \mathrm{~mm}$ ) was pushed into the same location. We performed an extra CT scan to confirm that the first coil was at the expected location, and measured the distance from the tip of the puncture needle to the visceral pleura on the CT image. Next, we pushed the second spring coil into the proper position, ensuring that the end of the second coil was closely combined with the first one. After fixing the needle core, the puncture was withdrawn from the puncture needle. We performed the final CT scan to observe whether the operation was successful and whether there were any complications. Similar to the Needle group, pneumonectomy was performed within 2 days of preoperative localization.

\section{Indicators}

The duration of localization, success rate, operation time, intraoperative bleeding, and positioning-related complications of patients were collected. We recorded the incidence of complications related to the success rate of the procedure through the imaging findings of the last $\mathrm{CT}$ and the intraoperative feedback from the surgeon, as well as other relevant indicators related to the procedure and clinical records. In order to control the influence of systematic errors on the experimental results, 100 patients were planned to be included in each group.

\section{Statistical analysis}

The data were recorded as $(\bar{x} \pm s)$ for normally-distributed data. For data with non-normal distribution, the MannWhitney rank sum test was employed with two independent samples. The Chi-squared test was used to compare counting data between the groups. Binary logistic regression was used to analyze the overall complications and related risk factors of each complication, and $\mathrm{P}<0.05$ was considered statistically significant. There was no loss to follow-up.

\section{Results}

About 300 patients have been recruited. After screening by reference to inclusion and exclusion criteria, we collected 100 cases of Needle group and 98 cases of Coil group. There were no statistical differences in age $(t=0.807$, $\mathrm{P}=0.420)$ and sex $\left(\chi^{2}=0.485, \mathrm{P}=0.486\right)$ between the two groups. No missing data for each variable of interest.

There were no significant differences in the diameter of the nodules $(\mathrm{Z}=-0.620, \mathrm{P}=0.536)$, distance from the edge of the nodules to the visceral pleura $(\mathrm{Z}=-0.649, \mathrm{P}=0.517)$, and depth of penetration $(\mathrm{Z}=-0.354, \mathrm{P}=0.724)$ between the Needle and Coil groups. Also, the resection ranges 

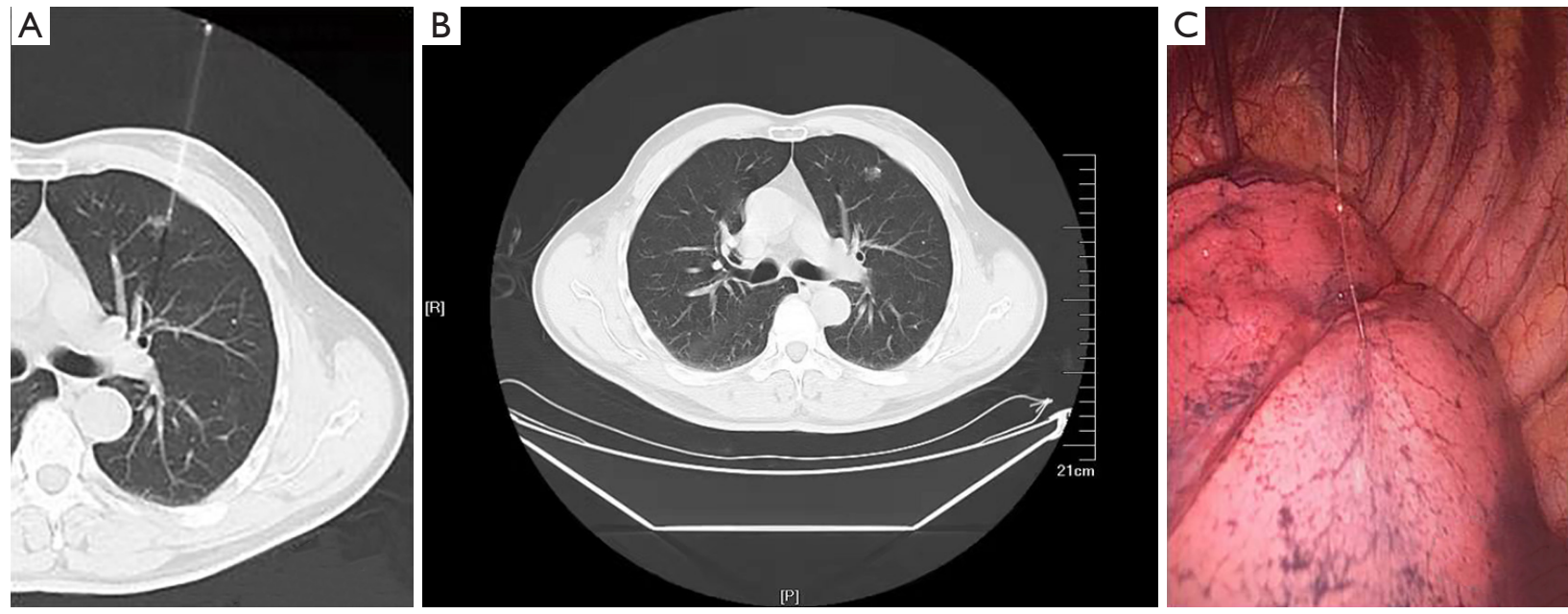

Figure 1 Located by the new coaxial needle, no complications. (A) The coaxial needle penetrated into chest; (B) completed preoperative localization; (C) the coaxial needle seen during operation.

Table 1 Comparison of positioning effect and operation time between the Needle and Coil groups $(\bar{x} \pm \mathrm{s})$

\begin{tabular}{lccc}
\hline Group & Success rate & Positioning time $(\min )$ & Operation time $(\mathrm{min})$ \\
\hline Needle group $(\mathrm{n}=100)$ & $99.00 \%$ & $15.27 \pm 4.60$ & $52.52 \pm 36.22$ \\
Coil group $(\mathrm{n}=98)$ & $97.96 \%$ & $18.54 \pm 3.71$ & $58.06 \pm 37.44$ \\
$\chi^{2} / Z$ & 0.157 & -5.099 & -2.984 \\
$P$ value & 1.000 & 0.001 & 0.003 \\
\hline
\end{tabular}

Table 2 Results of the $\chi^{2}$-test between the Needle and Coil groups for complications

\begin{tabular}{lccc}
\hline Group & Pneumothorax, $\mathrm{n}(\%)$ & Pneumorrhagia, $\mathrm{n}(\%)$ & Pleural reaction, $\mathrm{n}(\%)$ \\
\hline Needle group $(\mathrm{n}=100)$ & $20(20.00)$ & $13(13.00)$ & $3(3.00)$ \\
Coil group $(\mathrm{n}=98)$ & $32(32.65)$ & $24(24.49)$ & $2(2.04)$ \\
$\chi^{2}$ & 4.092 & 4.300 & 0.000 \\
$P$ value & 0.043 & 0.038 & 1.000 \\
\hline
\end{tabular}

(including pulmonary wedge resection and pulmonary lobectomy) of these two groups were similar $\left(\chi^{2}=0.163\right.$, $\mathrm{P}=0.686$ ).

In Needle group, the localization success rate is $99 \%$, the average localization operation time is $15.27 \pm 4.60 \mathrm{~min}$ (for example, Figure $1 A, B, C)$. As for Coil group, the localization success rate is $97.96 \%$, the average localization operation time is $18.54 \pm 3.71 \mathrm{~min}$. It seemed that, a majority of cases in both groups completed the positioning and operation. However, the Needle group had a significant advantage in terms of shortening the duration of both the positioning and the operation (Table 1).

The incidence of pneumothorax and pneumorrhagia in the Needle group were significantly lower than that in the Coil group (Table 2). As far as the further personalized analysis of each case, it is worth noting that some cases of pneumothorax were actually "pseudo-pneumothorax" caused by the positioning line pushing against the lung tissue (Figure 2A). Similarly, some cases of pneumorrhagia did not require additional treatment, as the second marked spring coil would immediately block the puncture passage (Figure 2B). 

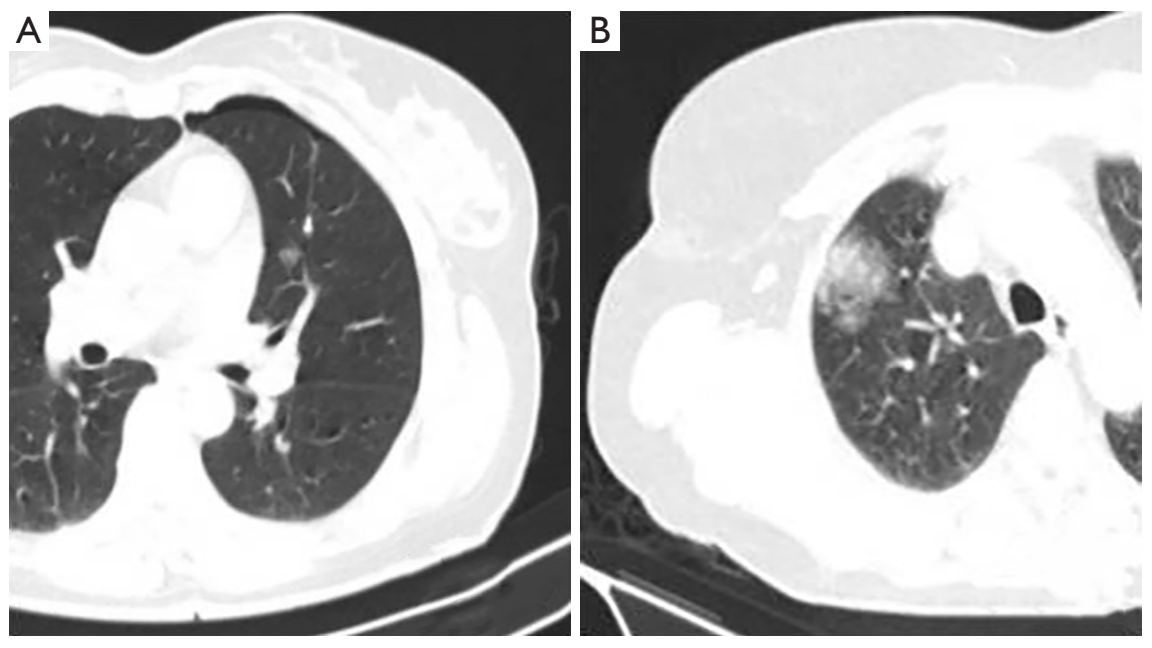

Figure 2 Mild pneumothorax and pneumorrhagia. (A) Pneumothorax linked with localization; (B) pneumorrhagia linked with localization.
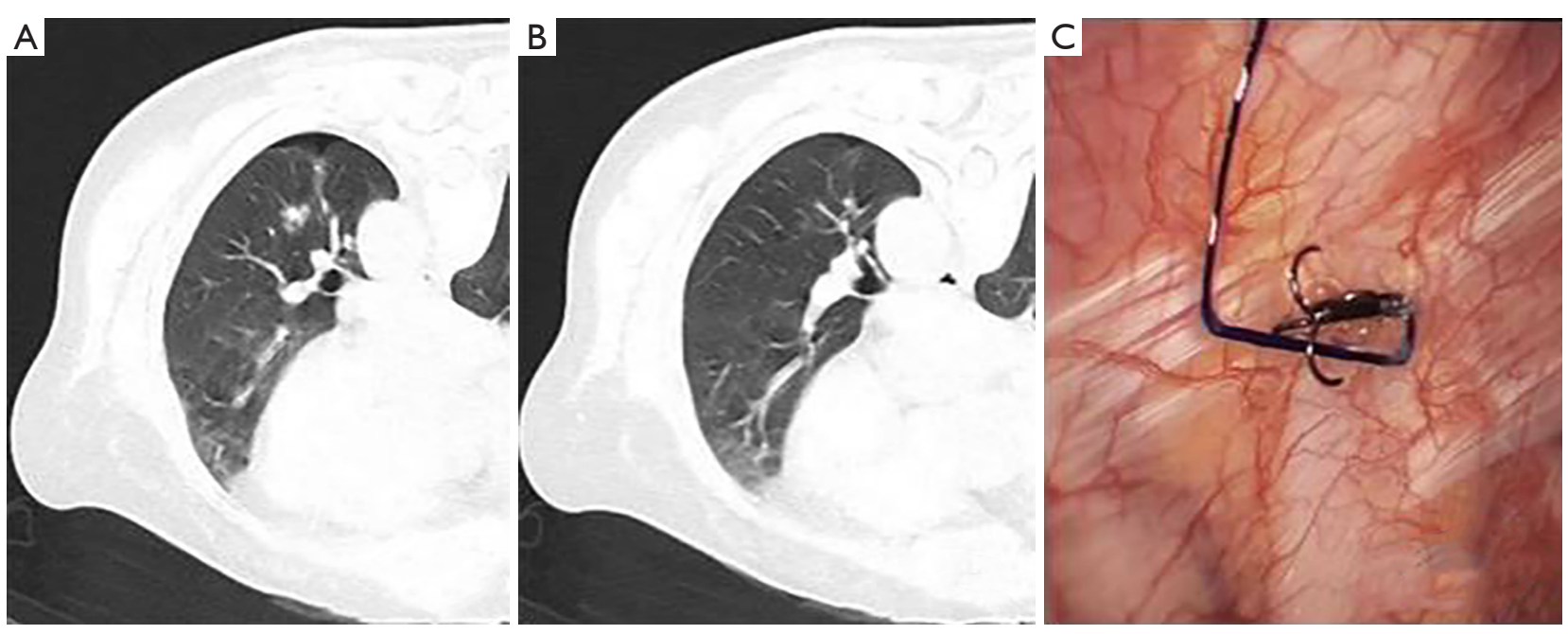

Figure 3 Three anchor pins lodged on the surface of the lung. (A,B) Characteristics shown on CT pictures; (C) the coaxial needle seen during operation. CT, computed tomography.

One case of failure occurred in the Needle group due to an error of device separation, which resulted in three hooks of the anchor lodged on the surface of the lung. However, one hook of the anchor needle was still visible next to the lesion during the operation, and the operation was performed successfully with needle guidance (Figure $3 A, B, C$ ).

As for the Coil group, one falling spring coil was close to the interlobar fissure, and the puncture needle did not completely enter the lung during the puncture, causing a partial fall-off during the release step (Figure 4A,B). Another case of failure occurred during the release process of the last coil, which shifted and fell off later during the patient's breathing (Figure 4C,D).

Logistic analysis indicated that the puncture depth was an independent risk factor for overall complications. Meanwhile, previous lung diseases and positioning time were independent risk factors for pneumothorax. As for pneumorrhagia, previous lung diseases, positioning time, and depth of penetration all increased the risk independently (Table 3).

\section{Discussion}

In recent years, VATS, as a minimally invasive thoracic 

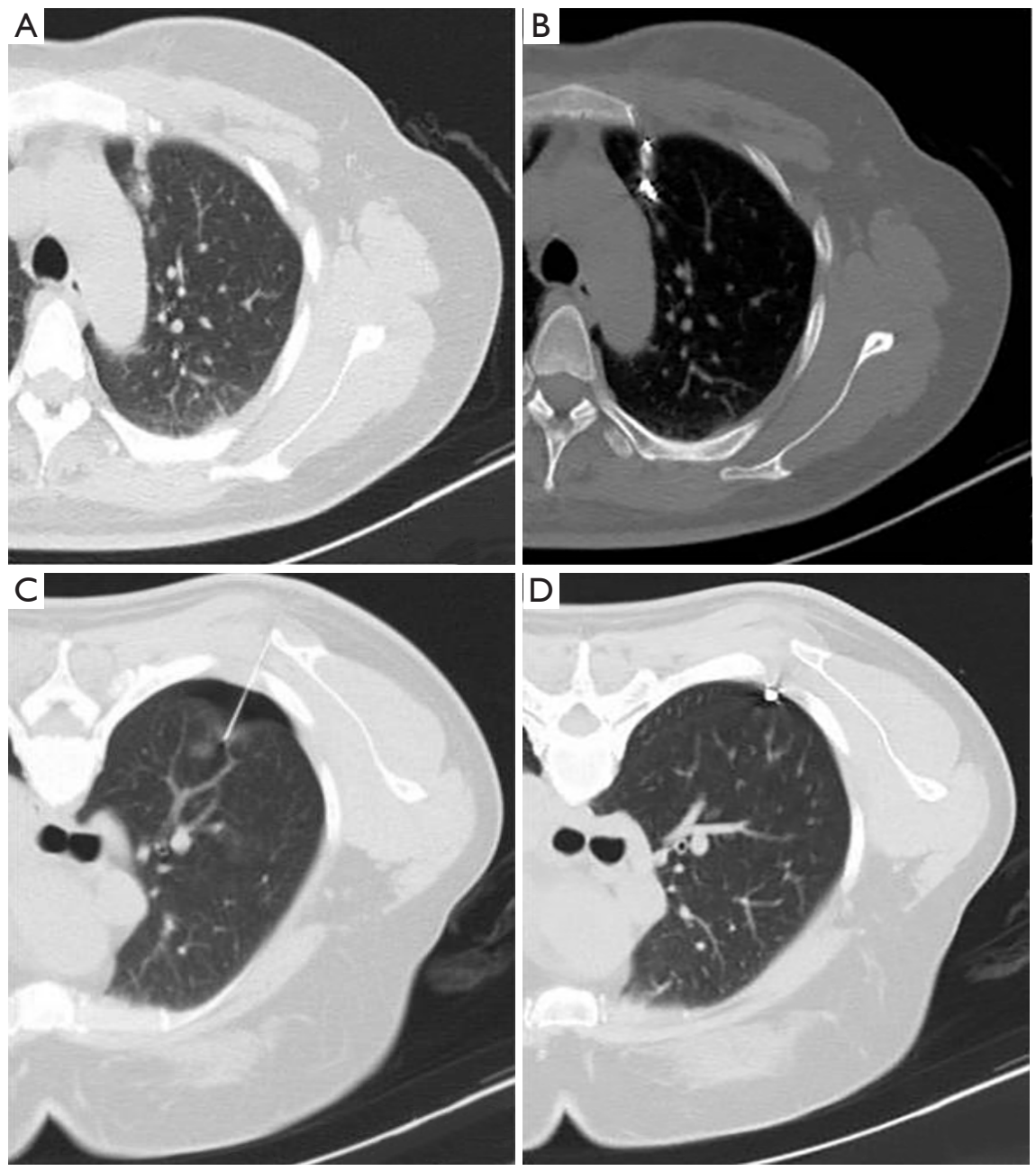

Figure 4 Unsuccessful preoperative localization. (A,B) The coaxial needle partly out of the lung; (C,D) the spring coil missing.

Table 3 Logistic analysis of factors linked with complications

\begin{tabular}{|c|c|c|c|c|c|c|}
\hline \multirow{2}{*}{ Influence factors } & \multicolumn{2}{|c|}{ Overall complications } & \multicolumn{2}{|c|}{ Pneumothorax } & \multicolumn{2}{|c|}{ Pneumorrhagia } \\
\hline & OR & $P$ value & OR & $P$ value & OR & $P$ value \\
\hline Gender & 1.533 & 0.193 & 1.064 & 0.851 & 2.075 & 0.082 \\
\hline Age & 0.979 & 0.170 & 0.988 & 0.447 & 0.975 & 0.195 \\
\hline Previous lung diseases & 2.618 & 0.004 & 2.397 & 0.008 & 2.418 & 0.034 \\
\hline Distance to visceral pleura & 0.989 & 0.615 & 0.971 & 0.205 & 1.015 & 0.569 \\
\hline Positioning time & 1.186 & 0.003 & 1.081 & 0.047 & 1.149 & 0.006 \\
\hline Depth of penetration & 2.382 & 0.013 & 1.054 & 0.345 & 1.229 & 0.003 \\
\hline
\end{tabular}

OR, odds ratio. 
surgery, has been increasingly used in the diagnosis and treatment of small-sized pulmonary tumor nodules $(\leq 2 \mathrm{~cm})(8)$. Effective preoperative localization can help thoracic surgeons and pathologists to quickly locate tumor nodules intraoperatively (9). Current location technology is varied, and includes liquid material positioning technology (methylene blue, iodine oil, medical glue, barium sulfate, radionuclide), solid material positioning technology, other positioning technologies (ultrasonic orientation and near infrared scan), and so on $(10,11)$. Each technology shows obvious advantages and disadvantages. Methylene blue staining is visible, but is readily diffused or metabolized. Patients who receive injections of either lipiodol or radionuclides produces radioactive contamination. It forces doctors to perform operations with protective gear. Ultrasound localization and infrared imaging both require the positioning of the radiologist on the operating table, and it requires special equipment. In this study, both of the two positioning methods can be completed 1-3 days before surgery in the interventional surgery room, saving the time of the surgeons and the radiologists, and making the plan of operation time more flexible. Although both localization methods required repeated CT radiation during a short period of time, we performed with low-dose CT, and patients did not require additional chest $\mathrm{CT}$ examination.

In this study, the double spring coil and new coaxial needle technologies were applied to preoperatively locate pulmonary nodules. The positioning success rate in the Needle group was $99.0 \%$, with only one case of failure occurring due to device detachment. In the Coil group, the positioning success rate was $98.0 \%$, with two cases of failure occurring due to dislocation of the spring coil. Undoubtedly, these two approaches are both able to complete most preoperative localization cases.

Complications presenting in the Needle group included 1 case of abscission (1.00\%), 13 cases of pulmonary hemorrhage (13.00\%), 20 cases of pneumothorax (20.00\%), and 3 cases of pleural reaction $(3.00 \%)$. Some cases of pneumothorax were actually "pseudo-pneumothorax" caused by the positioning line pushing against the lung tissue. In the cases which were showed in Figure 3A,B,C, the three angle of needle positioning anchored on the surface of lung, leading to a failure of positioning. We found that the anchor positioning needle was pushed along a $5 \mathrm{~mm}$ chute from the tip of needle. So, for the nodules that are approximately $5 \mathrm{~mm}$ from the pleural cavity, we released the anchor positioning needle near the chest wall, resulting in an attachment on the surface of lung. We then pushed the needle to the location of $5 \mathrm{~mm}$ from the heart-side of the nodule, and subsequently released the anchor pins. Using this procedure, the anchor needle no longer attached on the surface of the lung. Also, the soft positioning line did not cause discomfort to patients, and reduced the risk of the positioning needle shifting or falling off during breathing and daily activity. The whole procedure underwent in the sterile environment of the interventional room, and remainder of the soft positioning line outsides was properly covered with gauze. Aseptic handling and low risk of infection allowed surgeons to perform surgery days later, resulting in greater flexibility of the surgery schedule.

In the Coil group, 2 cases $(2.04 \%)$ of spring coil shedding, 32 cases $(32.65 \%)$ of pneumothorax, 24 cases (24.49\%) of pulmonary hemorrhage, and 2 cases $(2.04 \%)$ of pleural reaction were observed. Also, there was one case of a falling spring coil that was close to the interlobar fissure, and the puncture needle did not completely enter the lung during the puncture, causing a partial fall-off during the release step. The other failure case occurred during the release process of the last coil; the upper side of the coil was stuck in the chest wall, and shifted and fell off later as a result of the patient's postoperative breathing movement. In the operation, the piercing process of puncture needle and the first spring coil were more likely to cause slight pneumothorax and pneumorrhagia, as one of the reasons for the higher complications after positioning. In cases where mild pneumothorax and pneumorrhagia appeared along the path of puncture needle, the second marked spring coil would immediately block the puncture passage. Relevant studies have shown that positioning-related complications may be a consequence of unskilled operation, excessively superficial lesions, exceedingly shallow depth of the spring coil release, and nodules that are hidden in the interlobar fissure $(7,11)$. Experienced and proficient operators have a better potential to complete the operation $(9,12)$. In other word, this procedures of localization requires a longer learning cycle than that for positioning with the new coaxial needle.

The new positioning needle technology exhibited the characteristics of high safety, short operation time, accurate positioning, high success rate of positioning, and fewer complications. Compared with the double spring coil approach, the new coaxial needle is simple to operate, and requires less from the operator, and thus, it is easy to popularize. However, the new locating needle also requires some improvements; the length of the needle was $100 \mathrm{~mm}$, which is sometimes too short to penetrate the trick muscle 
tissue or the lung. We did not have access to longer punctual needle, nor did we see much coverage of them.

\section{Conclusions}

Compared with the double spring coil approach, the new coaxial needle provides high safety, short operation time, accurate positioning, high success rate of positioning, and fewer complications. It is also simple to operate and requires less from the operator, and thus, it is easy to popularize.

\section{Acknowledgments}

Funding: The Science and Technology Project of Health of Yunnan Province (2017NS254).

\section{Footnote}

Reporting Checklist: The authors have completed the STROBE reporting checklist. Available at https://dx.doi. org/10.21037/jtd-21-984

Data Sharing Statement: Available at https://dx.doi. org/10.21037/jtd-21-984

Conflicts of Interest: All authors have completed the ICMJE uniform disclosure form (available at https://dx.doi. org/10.21037/jtd-21-984). The authors have no conflicts of interest to declare.

Ethical Statement: The authors are accountable for all aspects of the work in ensuring that questions related to the accuracy or integrity of any part of the work are appropriately investigated and resolved. Ethical approval for the study was granted by the Ethics Review Committee of the First People's Hospital of Yunnan Province. Also, written informed consent was obtained from all patients before surgery. The study was conducted in accordance with the Declaration of Helsinki (as revised in 2013).

Open Access Statement: This is an Open Access article distributed in accordance with the Creative Commons Attribution-NonCommercial-NoDerivs 4.0 International License (CC BY-NC-ND 4.0), which permits the noncommercial replication and distribution of the article with the strict proviso that no changes or edits are made and the original work is properly cited (including links to both the formal publication through the relevant DOI and the license).
See: https://creativecommons.org/licenses/by-nc-nd/4.0/.

\section{References}

1. Yoshida R, Yoshizako T, Tanaka S, et al. CT-guided color marking of impalpable pulmonary nodules prior to video-assisted thoracoscopic surgery. Clin Imaging 2021;74:84-8.

2. Yang W, Sun W, Li Q, et al. Diagnostic accuracy of CTguided transthoracic needle biopsy for solitary pulmonary nodules. PLoS One 2015;10:e0131373.

3. Shen Y, Xu F, Zhu W, et al. Multiclassifier fusion based on radiomics features for the prediction of benign and malignant primary pulmonary solid nodules. Ann Transl Med 2020;8:171.

4. Su TH, Fan YF, Jin L, et al. CT-guided localization of small pulmonary nodules using adjacent microcoil implantation prior to video-assisted thoracoscopic surgical resection. Eur Radiol 2015;25:2627-33.

5. Shi $Z$, Chen C, Jiang S, et al. Uniportal video-assisted thoracic surgery resection of small ground-glass opacities (GGOs) localized with CT-guided placement of microcoils and palpation. J Thorac Dis 2016;8:1837-40.

6. Liu B, Gu C. Expert consensus workshop report: guidelines for preoperative assisted localization of small pulmonary nodules. J Cancer Res Ther 2020;16:967-73.

7. Kong J, Guo J, Zhang H, et al. CT-guided localization techniques of small pulmonary nodules: a prospective non-randomized controlled study on pulmonary nodule localization needle and methylene blue staining with surgical glue. J Thorac Dis 2020;12:6826-35.

8. Chen J, Pan X, Gu C, et al. The feasibility of navigation bronchoscopy-guided pulmonary microcoil localization of small pulmonary nodules prior to thoracoscopic surgery. Transl Lung Cancer Res 2020;9:2380-90.

9. Iqbal SI, Molgaard C, Williamson C, et al. Purposeful creation of a pneumothorax and chest tube placement to facilitate CT-guided coil localization of lung nodules before video-assisted thoracoscopic surgical wedge resection. J Vasc Interv Radiol 2014;25:1133-8.

10. Liu L, Zhang LJ, Chen B, et al. Novel CT-guided coil localization of peripheral pulmonary nodules prior to video-assisted thoracoscopic surgery: a pilot study. Acta Radiol 2014;55:699-706.

11. Almousa O, Mayo J, English J, et al. Microcoil-guided video-assisted thoracoscopic excision of nodules suspicious for metastasis in patients with extra- 
thoracic malignancies. Semin Thorac Cardiovasc Surg 2019;31:129-34.

12. Torre M, Ferraroli GM, Vanzulli A, et al. A new safe and stable spiral wire needle for thoracoscopic resection of

Cite this article as: Chen ZM, Xu JY, Cai WQ, Liao FC, Huo SQ, Yang JW, Peng J. The 4-hook anchor coaxial needle with scaled suture is superior to the double spring coil for preoperative localization. J Thorac Dis 2021;13(7):4455-4463. doi: $10.21037 /$ jtd-21-984 lung nodules. Chest 2004;125:2289-93.

(English Language Editor: A. Kassem) 